\title{
Physiological Regulation and Disorders of Phosphate Metabolism -Pivotal Role of Fibroblast Growth Factor 23-
}

\author{
Seiji Fukumoto
}

\begin{abstract}
Fibroblast growth factor (FGF) 23 has been identified as the last member of FGF family. FGF23 reduces serum phosphate level by suppressing proximal tubular phosphate reabsorption and intestinal phosphate absorption. FGF23 is produced by bone and acts on the kidney through a specific receptor system which is composed of Klotho and certain subtypes of FGF receptors. Excess actions of FGF23 cause several hypophosphatemic diseases characterized by impaired renal phosphate reabsorption and rickets/osteomalacia. In contrast, deficient actions of FGF23 result in hyperphosphatemic tumoral calcinosis with enhanced renal phosphate reabsorption. These results indicate that FGF23 works as a hormone to regulate the serum phosphate level.
\end{abstract}

Key words: hypophosphatemia, hyperphosphatemia, fibroblast growth factor, Klotho, hormone

(Inter Med 47: 337-343, 2008)

(DOI: 10.2169/internalmedicine.47.0730)

\section{Introduction}

Inorganic phosphate is an essential and the most abundant anion in our body. Phosphate is one of the major components of bone as hydroxyapatite $\left[\mathrm{Ca}_{10}\left(\mathrm{PO}_{4}\right)_{6}(\mathrm{OH})_{2}\right]$, a constituent of the cell membrane and works in various cellular functions as numerous phosphorylated metabolites. In order to accomplish these functions, it seems to be necessary that the circulatory phosphate level is regulated to within a narrow range. While the regulatory mechanisms of the serum calcium level have been extensively studied and explained by actions of two calcium-regulating hormones, parathyroid hormone $(\mathrm{PTH})$ and 1,25-dihydroxyvitamin $\mathrm{D}\left[1,25(\mathrm{OH})_{2} \mathrm{D}\right]$, it has been largely unknown how the serum phosphate level is maintained. Recent identification of fibroblast growth factor (FGF) 23 indicated that the serum phosphate level is regulated by a humoral mechanism and FGF23 plays an essential role in the maintenance of the serum phosphate level. In addition, several disorders of phosphate metabolism have been shown to be caused by deranged actions of FGF23.

\section{Regulation of the Serum Phosphate Level}

The serum phosphate level is regulated by intestinal phosphate absorption, renal phosphate reabsorption and dynamic equilibrium between extracellular phosphate and intracellular phosphate or phosphate in bone (Fig. 1). Of these, renal phosphate reabsorption is believed to be the main determinant of serum phosphate level at least in a chronic state, while acute changes of serum phosphate are usually caused by movement of extracellular phosphate into intracellular pool and bone, or vice versa. Filtered phosphate from glomeruli is largely reabsorbed in proximal tubules. Therefore, the molecule that mediates proximal tubular phosphate reabsorption has an indispensable role in the regulation of serum phosphate level.

Several sodium-dependent phosphate cotransporters have been identified to date. Type IIa and IIc sodium-phosphate cotransporters are expressed in proximal tubules and mediate physiological phosphate reabsorption in proximal tubules. Actually, knockout mice for type IIa sodium-phosphate cotransporter show hypophosphatemia by impaired reabsorp- 
tion of phosphate (1). In addition, mutations in a gene coding for type IIc sodium-phosphate cotransporter were shown to result in a genetic disease called hereditary hypophosphatemic rickets with hypercalciuria $(\mathrm{HHRH})(2,3)$. HHRH is characterized by hypophosphatemia due to impaired phosphate reabsorption. Because hypophosphatemia increases serum $1,25(\mathrm{OH})_{2} \mathrm{D}$ level and $1,25(\mathrm{OH})_{2} \mathrm{D}$ stimulates intestinal calcium absorption, patients with HHRH show high 1,25 $(\mathrm{OH})_{2} \mathrm{D}$ and hypercalciuria.

\section{Causes of Rickets/Osteomalacia}

Rickets and osteomalacia are bone diseases characterized by impaired mineralization of bone matrix. Because of this disordered mineralization, softening of bone is observed. Rickets is a disease of childhood which causes growth retardation and bowing of legs. On the contrary, muscle weakness and bone pain are major symptoms of osteomalacia observed in adults. Although there are various etiologies for rickets/osteomalacia, chronic hypophosphatemia is one of the most important and frequent causes of these diseases. There are several diseases causing hypophosphatemic rickets/osteomalacia which have very similar clinical features, these are three genetic diseases called autosomal dominant hypophosphatemic rickets/osteomalacia (ADHR), autosomal recessive hypophosphatemic rickets/osteomalacia (ARHR),

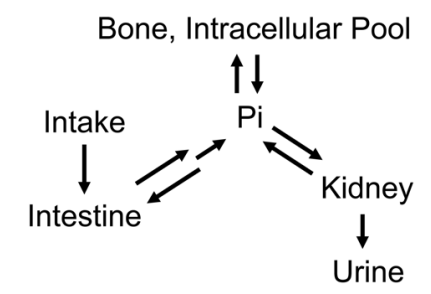

Figure 1. Phosphate balance. Serum phosphate level is regulated by intestinal phosphate absorption, renal phosphate reabsorption and dynamic equilibrium between extracellular phosphate and intracellular phosphate or phosphate in bone.
X-linked hypophosphatemic rickets/osteomalacia (XLH), one paraneoplastic syndrome named tumor-induced rickets/osteomalacia (TIO) and hypophosphatemic rickets/osteomalacia associated with McCune-Albright syndrome/fibrous dysplasia of bone. Hypophosphatemia due to impaired renal phosphate reabsorption is a typical feature of these diseases as seen in HHRH. However, serum $1,25(\mathrm{OH})_{2} \mathrm{D}$ level is not high, and remains low normal or low in patients with these diseases in contrast to high $1,25(\mathrm{HO})_{2} \mathrm{D}$ in HHRH. Therefore, these patients show inappropriately low $1,25(\mathrm{OH})_{2} \mathrm{D}$ level for hypophosphatemia indicating that both phosphate reabsorption and vitamin D metabolism are deranged in these diseases.

FGF23 was identified almost simultaneously by three groups. FGF23 was first cloned by homology to FGF15 in mice (4). In addition, positional cloning identified $F G F 23$ as a responsible gene for ADHR (5). Furthermore, FGF23 was cloned as a causative factor for TIO (6). These results indicate that FGF23 is involved in the development of several hypophosphatemic diseases.

\section{Structure of FGF23}

Sequence of cDNA for FGF23 indicates that FGF23 is a protein with 251 amino acids (Fig. 2). There is a signal peptide with 24 amino acids in the N-terminal portion of FGF 23 protein. Expression of recombinant FGF23 protein and detection of FGF23 protein by Western blotting indicated that a part of FGF23 protein is proteolytically cleaved between $\operatorname{Arg}^{179}$ and $\operatorname{Ser}^{180}$ by propotein convertase, furin $(6,7)$. FGF23 has a FGF homology region in the N-terminal portion of this processing site.

FGF23 belongs to FGF family. FGF family members are defined as humoral factors which have a FGF homology region and show affinity for FGF receptors (FGFRs) (8). There are 22 FGF family members in humans and these family members are subdivided into several subfamilies. FGF23 belongs to FGF19 subfamily together with FGF19

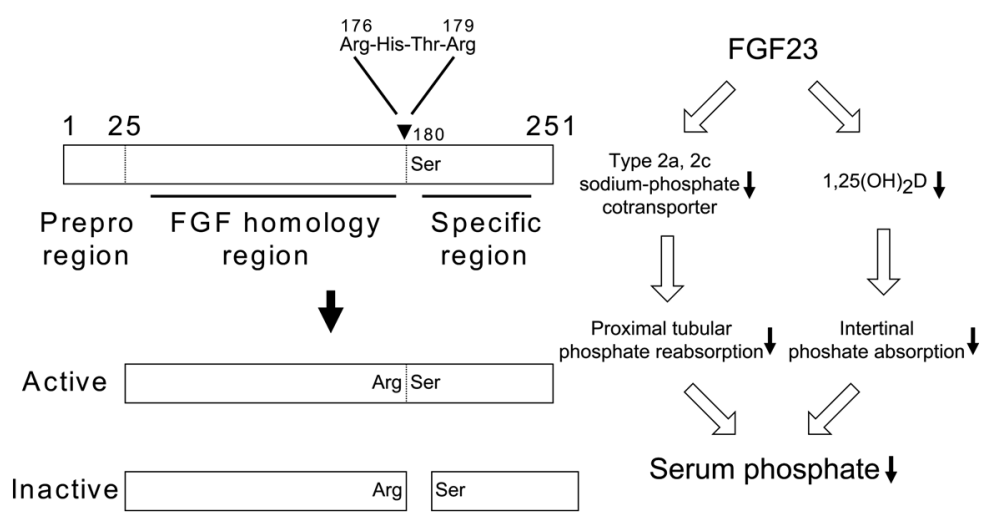

Figure 2. Structure and function of FGF23. FGF23 is a protein with 251 amino acids. There is a signal peptide with 24 amino acids in the N-terminal portion of FGF23 protein. A part of FGF23 is cleaved between $\mathrm{Arg}^{179}$ and $\mathrm{Ser}^{180}$ by furin recognizing $\mathrm{Arg}^{176}$-X-X-Arg ${ }^{179}$ motif. FGF23 has a FGF homology region in the $\mathrm{N}$-terminal portion of this processing site. FGF23 reduces serum phosphate by suppressing proximal tubular phosphate reabsorption and intestinal phosphate absorption. 
and FGF21. Four FGF11 subfamily members, FGF11 to FGF14, are also called FGF homologous factors (9). Because FGF11 subfamily members show no affinity for FGFRs, some researchers exclude these members from the FGF family.

\section{Actions of FGF23}

The biological activity of FGF23 was examined using recombinant FGF23. A single injection of FGF23 resulted in reduced serum phosphate and $1,25(\mathrm{OH})_{2} \mathrm{D}$ levels (10). FGF 23 reduces renal phosphate reabsorption by suppressing expression of type $2 \mathrm{a}$ and $2 \mathrm{c}$ sodium-phosphate cotransporter in brush border membrane of proximal tubules. Prior to the reduction of serum phosphate level, FGF23 suppresses the expression of 25-hydroxyvitamin D-1 $\alpha$-hydroxylase and conversely enhances the expression of 25-hydroxyvitamin D-24-hydroxylase in kidney. 25-hydroxyvitamin D-1 $\alpha$ hydroxylase is an enzyme that mediates the production of $1,25(\mathrm{OH})_{2} \mathrm{D}$ and 25-hydroxyvitamin D-24-hydroxylase converts $1,25(\mathrm{OH})_{2} \mathrm{D}$ into more hydrophilic metabolites with less activity. Therefore, FGF23 reduces serum $1,25(\mathrm{OH})_{2} \mathrm{D}$ by modifying expression levels of these vitamin Dmetabolizing enzymes. Because $1,25(\mathrm{OH})_{2} \mathrm{D}$ stimulates intestinal phosphate absorption, FGF23 reduces serum phosphate by suppressing proximal tubular phosphate reabsorption and intestinal phosphate absorption (Fig. 2).

This biological activity of FGF23 to reduce serum phosphate level was observed using uncleaved FGF23. In contrast, processed $\mathrm{N}$-terminal and $\mathrm{C}$-terminal fragments of FGF23 did not reduce the serum phosphate level (11). These results indicate that the processing of FGF23 protein between $\operatorname{Arg}^{179}$ and $\operatorname{Ser}^{180}$ abolishes its effect to reduce serum phosphate level (Fig. 2).

FGF23 knockout mice were created to examine whether FGF23 has physiological functions $(12,13)$. These mice show hyperphosphatemia, enhanced renal phosphate reabsorption and high $1,25(\mathrm{OH})_{2} \mathrm{D}$ level. These results indicate that FGF23 is physiologically working to reduce serum phosphate and $1,25(\mathrm{OH})_{2} \mathrm{D}$ levels. Replacement of $F G F 23$ gene by lacZ indicated that FGF23 is produced by bone (13). Other study shows that FGF23 is produced by osteocytes (14). Therefore, FGF23 seems to be produced by bone and regulate serum phosphate and $1,25(\mathrm{OH})_{2} \mathrm{D}$ levels acting on kidney. Thus, there should be a specific receptor for FGF 23 in kidney. These features are clearly different from those of "classical" FGF family members. Classical FGF family members have been shown to bind to FGFRs and thought to work as local factors.

In order to identify the specific receptor for FGF23 in kidney, binding proteins to FGF23 in renal homogenate was analyzed and the major binding protein was found to be Klotho (15). Klotho mice were created by transgenic method and these mice show severely reduced expression of Klotho (16). Klotho mice have been regarded as a model animal for senescence. However, it has been also shown that Klotho mice have hyperphosphatemia and high $1,25(\mathrm{OH})_{2} \mathrm{D}$ levels like FGF23 knockout mice suggesting the involvement of Klotho in FGF23 signaling (16). Subsequent in vitro studies indicated that FGF23 can provoke intracellular signaling such as phosphorylation of extracellular signal-regulated kinase and induction of early growth response-1 only in cells expressing Klotho (15). However, Klotho alone was not sufficient for FGF23 signaling. There are four genes for FGFRs and alternate splicing from these genes produces several subtypes of FGFRs. Klotho was shown to work as a coreceptor for FGF23 together with certain subtypes of FGFRs including FGFR1c $(15,17)$. Thus, FGF23 is produced by bone, works on kidney through a specific receptor for FGF23 and physiologically regulates serum phosphate and $1,25(\mathrm{OH})_{2} \mathrm{D}$ levels. These features indicate that FGF23 is a hormone rather than a local cytokine like other classical members of FGF family (18).

\section{Involvement of FGF23 in the Development of Hypophosphatemic Rickets/Osteomalacia}

As mentioned before, ADHR, ARHR, XLH, TIO and hypophosphatemic rickets/osteomalacia associated with McCune-Albright syndrome/fibrous dysplasia are characterized by hypophosphatemia and rather low $1,25(\mathrm{OH})_{2} \mathrm{D}$ levels. These biochemical features are those expected to be caused by excess actions of FGF23. Actually, after the cloning of $F G F 23$, several enzyme-linked immunosorbent assays for FGF23 have been established. These assays confirmed that FGF23 levels are basically high in patients with these hypophosphatemic diseases $(19,20)$. However, the mechanisms of excess actions of FGF23 in these diseases are variable (Table 1).

$F G F 23$ was cloned from a tumor responsible for TIO (6). This disease has a profound effect on the quality of life of the patients. Because of severe muscle weakness and bone pain, patients with TIO sometimes become completely bedridden. However, this disease is cured by complete resection of responsible tumors. Circulatory FGF23 level is elevated in virtually all patients with TIO, and rapidly decreases into or below the reference range after removal of the responsible tumors (20-22). In addition, it has been shown that tumors causing TIO strongly express FGF23 $(6,23)$. Therefore, TIO is considered to be caused by overexpression of FGF23 in responsible tumors. Most tumors causing TIO are now pathologically classified as phosphaturic mesenchymal tumor (mixed connective tissue variant) (PMTMCT) (24). However, it is unknown why some PMTMCTs overexpress FGF23.

FGF23 was also cloned as a responsible gene for ADHR and several missense mutations in FGF23 gene were reported in patients with FGF23 (5). These mutations are found in codons coding for either $\operatorname{Arg}^{176}$ or $\operatorname{Arg}^{179}$ just before the processing site between $\operatorname{Arg}^{179}$ and $\operatorname{Ser}^{180}$ (Fig. 2). The amino acid sequence before the processing site, $\mathrm{Arg}^{176}-\mathrm{X}-\mathrm{X}$ $\operatorname{Arg}^{179}$, is the consensus site recognized by furin. Therefore, 
Table 1. Hypophosphatemic Diseases Caused by Excess Actions of FGF23 and Causes of Excess FGF23 Action

\author{
Disease \\ Autosomal dominant hypophosphatemic rickets/osteomalacia (ADHR) \\ Autosomal recessive hypophosphatemic rickets/osteomalacia (ARHR) \\ $\mathrm{X}$-linked hypophosphatemic rickets/osteomalacia (XLH) \\ McCune-Albright syndrome/Fibrous dysplasia \\ Tumor-induced rickets/osteomalacia
}

\author{
Cause of excess FGF23 action \\ Dysregulated expression of FGF23 by mutations in FGF23 gene \\ Overexpression of FGF23 in bone by mutations in DMP1 gene \\ Overexpression of FGF23 in bone by mutations in PEHX gene \\ Overexpression of FGF23 in bone \\ Overexpression of FGF23 in responsible tumors
}

PHEX: phosphate-regulating gene with homologies to endopeptidases on the X chromosome

DMP1: dentin matrix protein 1

mutations in $\mathrm{Arg}^{176}$ or $\mathrm{Arg}^{179}$ destroy this consensus sequence and were considered to result in impaired processing of FGF 23 protein. Actually, mutant FGF23 proteins observed in patients with ADHR are resistant to the processing between $\operatorname{Arg}^{179}$ and $\operatorname{Ser}^{180}(11,25)$. This resistance to the processing was expected to cause increased amount of biologically active full-length FGF23 and enhanced activity of FGF23. However, FGF23 level is not always high in patients with ADHR. FGF23 level fluctuates in these patients and it was reported that high FGF23 level is associated with hypophosphatemia (26). These results suggest that mutations in FGF 23 gene somehow impair the regulatory mechanism of FGF 23 production and dysregulated FGF23 production underlie hypophosphatemia in patients with ADHR.

Responsible genes for XLH and ARHR are phosphateregulating gene with homologies to endopeptidases on the $X$ chromosome (PHEX) (27) and dentin matrix protein 1 (DMP 1) $(28,29)$, respectively. Although the precise functions of PHEX and DMP1 proteins are not yet elucidated, enhanced expression of FGF23 in bone has been reported in model mice for XLH and DMP1-null mice $(28,30)$. Together with elevated FGF23 levels in patients with these diseases (19-21, 28), it is suggested that PHEX and DMP1 somehow suppress FGF23 expression in bone. FGF23 has been also shown to be overexpressed in bone including regions affected by fibrous dysplasia in patients with McCuneAlbright syndrome/fibrous dysplasia (31). While somatic activating mutations in GNAS1 gene are responsible for this disease, it is unclear whether or not increased cyclic AMP level causes enhanced expression of FGF23. Regulatory mechanisms of FGF23 expression in normal and abnormal bone tissues are one of important remaining questions to be clarified.

\section{FGF23 and Hyperphosphatemic Diseases}

In contrast to several hypophosphatemic diseases caused by excess actions of FGF23, patients with hyperphosphatemic tumoral calcinosis show hyperphosphatemia, en- hanced renal tubular phosphate reabsorption and a high 1,25 $(\mathrm{OH})_{2} \mathrm{D}$ level (32). These features are also observed in $F G F$ 23 knockout mice and Klotho mice. Thus, it was postulated that hyperphosphatemic tumoral calcinosis is caused by deficient actions of FGF23. Therefore, it was a surprise that GALNT3 encoding UDP-N-acetyl-alpha-D-galactosamine: polypeptide $\mathrm{N}$-acetylgalactosaminyltransferase 3 (ppGaNTase-T3) was first reported to be responsible for hyperphosphatemic tumoral calcinosis (33). ppGaNTase-T3 is an enzyme that transfers $\mathrm{N}$-acetylgalactosamine from UDP-N-acetyl-alphaD-galactosamine to serine or threonine residue as an initial sugar of mucin-type O-linked glycosylation. However, it was unclear how mutations in GALNT3 gene cause hyperphosphatemia. There are several assay methods for FGF23. Intact assay uses two monoclonal antibodies that recognize $\mathrm{N}$ terminal and C-terminal potion of the processing site of FGF23 and detects only full-length uncleaved FGF23 (20). On the other hand, C-terminal assay uses two kinds of polyclonal antibodies to the $\mathrm{C}$-terminal region of the processing site and measures both full-length and cleaved C-terminal fragment of FGF23 (19). FGF23 levels evaluated by these two assays correlate very well in patients with XLH (21). However, when FGF23 levels in patients with GALNT3 mutations were analyzed by these two assays, it was found that FGF23 was quite high by C-terminal assay, but low-low normal in intact assay (34) (Table 2). These results suggested that there is only a little full-length FGF23, but large amount of processed C-terminal fragment of FGF23 in these patients. Actually, in vitro study indicated that FGF23 has three O-linked glycans (34) and ppGaNTase-T3 is specifically involved in the synthesis of O-glycan attaching to $\mathrm{Thr}^{178}$ just before the processing site between $\mathrm{Arg}^{179}$ and $\operatorname{Ser}^{180}$ (35). In addition, silencing ppGaNTase-T3 expression enhanced the processing of FGF23 protein (34). Therefore, the clinical features of patients with mutations in GALNT3 gene could be explained as shown below. Mutations in GALNT3 gene result in impaired glycosylation of FGF23 proteins at $\mathrm{Thr}^{178}$ and cause enhanced processing of FGF23. Hyperphosphatemia and high $1,25(\mathrm{OH})_{2} \mathrm{D}$ derive from defi- 
Table 2. Hyperphosphatemic Tumoral Calcinosis and Circulatory FGF23 Levels

Causative gene Mechanism of impaired FGF23 action

FGF23

GALNT3

Enhanced processing of FGF23

Klotho

Resistance to FGF23

cient actions of full-length FGF23. Although the precise mechanism of FGF23 production is not clear at the moment, hyperphosphatemia, high $1,25(\mathrm{OH})_{2} \mathrm{D}$ or other metabolic changes stimulate FGF23 production. Together with enhanced processing of FGF23 protein, overexpression of FGF 23 results in high C-terminal fragment of FGF23.

Subsequently, mutations in FGF23 gene were also shown to cause hyperphosphatemic tumoral calcinosis (36-38). FGF 23 levels in patients with mutations in FGF23 are essentially the same as those with mutations in GALNT3, extremely high by C-terminal assay and low by full-length assay (36) (Table 2). Several missense mutations of FGF23 gene were reported and these mutant FGF23 proteins are somehow susceptible to the processing. The mechanism of this susceptibility to the processing is unclear at the moment.

Recent analysis indicated that hyperphosphatemic tumoral calcinosis is also caused by a mutation in Klotho gene again indicating the involvement of Klotho in FGF23 signaling (39). FGF23 level in this patient is very high both by intact and C-terminal assays (Table 2). These results indicate that insensitivity to FGF23 is the cause of hyperphosphatemia in this patient in contrast to enhanced processing of FGF23 protein in patients with mutations in GALNT3 or FGF23. Thus, hyperphosphatemic tumoral calcinosis by a mutation in Klotho gene is another example of hormone resistance.

FGF23 was reported to be high in hyperphosphatemic patients by chronic renal failure (40-43) or hypoparathyroidism (44). Considering the biological activities of FGF23, this high FGF23 seems to be the result of hyperphosphatemia again suggesting that hyperphosphatemia enhances FGF23 expression. However, FGF23 is extremely high in some patients with chronic renal failure undergoing dialysis in contrast to a slight increase of FGF23 in patients with hypoparathyroidism. These results suggest that some metabolic changes in end-stage renal disease stimulate FGF23 production although the precise mechanism of this high FGF23 is unknown.
FGF23 levels

$$
\text { Intact assay C-terminal assay }
$$

Low - low normal High

Low - low normal High

High High

\section{Summary and Future Perspectives}

Studies in the past several years clarified the essential role of FGF23 as a hormone to regulate serum phosphate and $1,25(\mathrm{OH})_{2} \mathrm{D}$ levels. In addition, several hypophosphatemic and hyperphosphatemic diseases were shown to be caused by deranged actions of FGF23. The identification of Klotho as a coreceptor for FGF23 showed the novel means of interaction between FGF family members and FGFRs, and indicated the possibility of similar ligand-receptor coupling. Actually, FGF19 produced by the intestine was shown to inhibit bile acid synthesis in the liver through FGFR4 and $\beta$ Klotho, a homologous protein to Klotho (45-48). Likewise, FGF21 mainly produced by the liver acts on adipose tissue through FGFR1c and $\beta$ Klotho $(47,49-51)$. Based on these results, FGF19 family members, FGF19, FGF21 and FGF 23, are now called endocrine FGFs.

Despite these findings, several important questions in addition to those already mentioned remain. First, while several results suggest that the serum phosphate level is at least one of the regulators of FGF23 production and circulatory FGF23 level, it is unknown how and where changes of serum phosphate are sensed in our body and induce metabolic changes leading to altered FGF23 level. Second, it is not clear how the processing of FGF23 protein is controlled and why the processing between $\operatorname{Arg}^{179}$ and $\operatorname{Ser}^{180}$ is necessary. Furthermore, intracellular signaling molecules beyond Klotho-FGFR1c complex induced by FGF23 are largely unknown. Future studies clarifying these issues will hopefully help to establish novel therapeutic measures for deranged phosphate and vitamin D metabolism.

\section{Acknowledgement}

This work was supported in part by grants from the Ministry of Education, Culture, Sports, Science and Technology, and from the Ministry of Health, Labour and Welfare, Japan. 


\section{References}

1. Beck L, Karaplis AC, Amizuka N, Hewson AS, Ozawa H, Tenenhouse HS. Targeted inactivation of Npt2 in mice leads to severe renal phosphate wasting, hypercalciuria, and skeletal abnormalities. Proc Natl Acad Sci U S A 95: 5372-5377, 1998.

2. Bergwitz C, Roslin NM, Tieder M, et al. SLC34A3 mutations in patients with hereditary hypophosphatemic rickets with hypercalciuria predict a key role for the sodium-phosphate cotransporter $\mathrm{NaPi}$-IIc in maintaining phosphate homeostasis. Am J Hum Genet 78: 179-192, 2006.

3. Lorenz-Depiereux B, Benet-Pages A, Eckstein G, et al. Hereditary hypophosphatemic rickets with hypercalciuria is caused by mutations in the sodium-phosphate cotransporter gene SLC34A3. Am J Hum Genet 78: 193-201, 2006.

4. Yamashita T, Yoshioka M, Itoh N. Identification of a novel fibroblast growth factor, FGF-23, preferentially expressed in the ventrolateral thalamic nucleus of the brain. Biochem Biophys Res Commun 277: 494-498, 2000.

5. ADHR Consortium. Autosomal dominant hypophosphataemic rickets is associated with mutations in FGF23. Nat Genet 26: 345$348,2000$.

6. Shimada T, Mizutani S, Muto T, et al. Cloning and characterization of FGF23 as a causative factor of tumor-induced osteomalacia. Proc Natl Acad Sci U S A 98: 6500-6505, 2001.

7. Benet-Pages A, Lorenz-Depiereux B, Zischka H, White KE, Econs MJ, Strom TM. FGF23 is processed by proprotein convertases but not by PHEX. Bone 35: 455-462, 2004.

8. Itoh N, Ornitz DM. Evolution of the Fgf and Fgfr gene families. Trends Genet 20: 563-569, 2004.

9. Olsen SK, Garbi M, Zampieri N, et al. Fibroblast growth factor (FGF) homologous factors share structural but not functional homology with FGFs. J Biol Chem 278: 34226-34236, 2003.

10. Shimada T, Hasegawa H, Yamazaki Y, et al. FGF-23 is a potent regulator of vitamin $\mathrm{D}$ metabolism and phosphate homeostasis. J Bone Miner Res 19: 429-435, 2004.

11. Shimada T, Muto T, Urakawa I, et al. Mutant FGF-23 responsible for autosomal dominant hypophosphatemic rickets is resistant to proteolytic cleavage and causes hypophosphatemia in vivo. Endocrinology 143: 3179-3182, 2002.

12. Shimada T, Kakitani M, Yamazaki $Y$, et al. Targeted ablation of Fgf23 demonstrates an essential physiological role of FGF-23 in phosphate and vitamin D metabolism. J Clin Invest 113: 561-568, 2004.

13. Sitara D, Razzaque MS, Hesse M, et al. Homozygous ablation of fibroblast growth factor-23 results in hyperphosphatemia and impaired skeletogenesis, and reverses hypophosphatemia in Phexdeficient mice. Matrix Biol 23: 421-432, 2004.

14. Liu S, Zhou J, Tang W, Jiang X, Rowe DW, Quarles LD. Pathogenic role of FGF23 in Hyp mice. Am J Physiol Endocrinol Metab 291: E38-E49, 2006.

15. Urakawa I, Yamazaki Y, Shimada T, et al. Klotho converts canonical FGF receptor into a specific receptor for FGF23. Nature 444: 770-774, 2006.

16. Kuro-o M, Matsumura $\mathrm{Y}$, Aizawa $\mathrm{H}$, et al. Mutation of the mouse klotho gene leads to a syndrome resembling ageing. Nature 390: 45-51, 1997.

17. Kurosu H, Ogawa Y, Miyoshi M, et al. Regulation of fibroblast growth factor-23 signaling by klotho. J Biol Chem 281: 61206123, 2006.

18. Fukumoto S, Yamashita T. FGF23 is a hormone regulating phosphate metabolism-Unique biological characteristics of FGF23-. Bone 40: 1190-1195, 2007.

19. Jonsson KB, Zahradnik R, Larsson $T$, et al. Fibroblast growth factor 23 in oncogenic osteomalacia and X-linked hypophosphatemia.
N Engl J Med 348: 1656-1663, 2003.

20. Yamazaki Y, Okazaki R, Shibata $M$, et al. Increased circulatory level of biologically active full-length FGF-23 in patients with hypophosphatemic rickets/osteomalacia. J Clin Endocrinol Metab 87: 4957-4960, 2002.

21. Ito N, Fukumoto $\mathrm{S}$, Takeuchi $\mathrm{Y}$, et al. Comparison of two assays for fibroblast growth factor (FGF)-23. J Bone Miner Metab 23: 435-440, 2005.

22. Takeuchi Y, Suzuki H, Ogura S, et al. Venous sampling for fibroblast growth factor-23 confirms preoperative diagnosis of tumorinduced osteomalacia. J Clin Endocrinol Metab 89: 3979-3982, 2004.

23. White KE, Jonsson KB, Carn G, et al. The autosomal dominant hypophosphatemic rickets (ADHR) gene is a secreted polypeptide overexpressed by tumors that cause phosphate wasting. J Clin Endocrinol Metab 86: 497-500, 2001.

24. Folpe AL, Fanburg-Smith JC, Billings SD, et al. Most osteomalacia-associated mesenchymal tumors are a single histopathologic entity: an analysis of 32 cases and a comprehensive review of the literature. Am J Surg Pathol 28: 1-30, 2004.

25. White KE, Carn G, Lorenz-Depiereux B, Benet-Pages A, Strom TM, Econs MJ. Autosomal-dominant hypophosphatemic rickets (ADHR) mutations stabilize FGF-23. Kidney Int 60: 2079-2086, 2001.

26. Imel EA, Hui SL, Econs MJ. FGF23 concentrations vary with disease status in autosomal dominant hypophosphatemic rickets. J Bone Miner Res 22: 520-526, 2007.

27. The HYP Consortium. A gene (PEX) with homologies to endopeptidases is mutated in patients with X-linked hypophosphatemic rickets. Nat Genet 11: 130-136, 1995.

28. Feng JQ, Ward LM, Liu S, et al. Loss of DMP1 causes rickets and osteomalacia and identifies a role for osteocytes in mineral metabolism. Nat Genet 38: 1310-1315, 2006.

29. Lorenz-Depiereux B, Bastepe M, Benet-Pages A, et al. DMP1 mutations in autosomal recessive hypophosphatemia implicate a bone matrix protein in the regulation of phosphate homeostasis. Nat Genet 38: 1248-1250, 2006.

30. Liu S, Guo R, Simpson LG, Xiao ZS, Burnham CE, Quarles LD. Regulation of fibroblastic growth factor 23 expression but not degradation by PHEX. J Biol Chem 278: 37419-37426, 2003.

31. Riminucci M, Collins MT, Fedarko NS, et al. FGF-23 in fibrous dysplasia of bone and its relationship to renal phosphate wasting. J Clin Invest 112: 683-692, 2003.

32. Lyles KW, Halsey DL, Friedman NE, Lobaugh B. Correlations of serum concentrations of 1,25-dihydroxyvitamin D, phosphorus, and parathyroid hormone in tumoral calcinosis. J Clin Endocrinol Metab 67: 88-92, 1988.

33. Topaz O, Shurman DL, Bergman R, et al. Mutations in GALNT3, encoding a protein involved in O-linked glycosylation, cause familial tumoral calcinosis. Nat Genet 36: 579-581, 2004.

34. Frishberg Y, Ito N, Rinat C, et al. Hyperostosis-hyperphosphatemia syndrome: A congenital disorder of O-glycosylation associated with augmented processing of fibroblast growth factor 23. J Bone Miner Res 22: 235-242, 2007.

35. Kato K, Jeanneau C, Tarp MA, et al. Polypeptide GalNActransferase T3 and familial tumoral calcinosis. Secretion of fibroblast growth factor 23 requires O-glycosylation. J Biol Chem 281: 18370-18377, 2006.

36. Araya K, Fukumoto S, Backenroth $\mathrm{R}$, et al. A novel mutation in fibroblast growth factor 23 gene as a cause of tumoral calcinosis. J Clin Endocrinol Metab 90: 5523-5527, 2005.

37. Benet-Pages A, Orlik P, Strom TM, Lorenz-Depiereux B. An FGF23 missense mutation causes familial tumoral calcinosis with 
hyperphosphatemia. Hum Mol Genet 14: 385-390, 2005.

38. Larsson T, Yu X, Davis SI, et al. A novel recessive mutation in fibroblast growth factor-23 causes familial tumoral calcinosis. J Clin Endocrinol Metab 90: 2424-2427, 2005.

39. Ichikawa S, Imel EA, Kreiter ML, et al. A homozygous missense mutation in human KLOTHO causes severe tumoral calcinosis. J Clin Invest 117: 2684-2691, 2007.

40. Imanishi Y, Inaba M, Nakatsuka K, et al. FGF-23 in patients with end-stage renal disease on hemodialysis. Kidney Int 65: 19431946, 2004.

41. Larsson $T$, Nisbeth $U$, Ljunggren O, Juppner H, Jonsson KB. Circulating concentration of FGF-23 increases as renal function declines in patients with chronic kidney disease, but does not change in response to variation in phosphate intake in healthy volunteers. Kidney Int 64: 2272-2279, 2003.

42. Shigematsu T, Kazama JJ, Yamashita T, et al. Possible involvement of circulating fibroblast growth factor 23 in the development of secondary hyperparathyroidism associated with renal insufficiency. Am J Kidney Dis 44: 250-256, 2004.

43. Weber TJ, Liu S, Indridason OS, Quarles LD. Serum FGF23 levels in normal and disordered phosphorus homeostasis. J Bone Miner Res 18: 1227-1234, 2003.

44. Gupta A, Winer K, Econs MJ, Marx SJ, Collins MT. FGF-23 is elevated by chronic hyperphosphatemia. J Clin Endocrinol Metab
89: 4489-4492, 2004.

45. Inagaki T, Choi M, Moschetta A, et al. Fibroblast growth factor 15 functions as an enterohepatic signal to regulate bile acid homeostasis. Cell Metab 2: 217-225, 2005.

46. Ito S, Fujimori T, Furuya A, Satoh J, Nabeshima Y, Nabeshima Y. Impaired negative feedback suppression of bile acid synthesis in mice lacking betaKlotho. J Clin Invest 115: 2202-2208, 2005.

47. Kurosu H, Choi M, Ogawa Y, et al. Tissue-specific expression of beta KLOTHO and fibroblast growth factor receptor isoforms determines metabolic activity of FGF19 and FGF21. J Biol Chem 282: 26687-26695, 2007.

48. Lin BC, Wang M, Blackmore C, Desnoyers LR. Liver specific activities of FGF19 require KLOTHO beta. J Biol Chem 282: 27277-27284, 2007.

49. Badman MK, Pissios P, Kennedy AR, Koukos G, Flier JS, Maratos-Flier E. Hepatic fibroblast growth factor 21 is regulated by PPARalpha and is a key mediator of hepatic lipid metabolism in ketotic states. Cell Metab 5: 426-437, 2007.

50. Inagaki T, Dutchak P, Zhao G, et al. Endocrine regulation of the fasting response by PPARalpha-mediated induction of fibroblast growth factor 21. Cell Metab 5: 415-425, 2007.

51. Ogawa Y, Kurosu H, Yamamoto M, et al. BetaKlotho is required for metabolic activity of fibroblast growth factor 21. Proc Natl Acad Sci U S A 104: 7432-7437, 2007.

(C) 2008 The Japanese Society of Internal Medicine http://www.naika.or.jp/imindex.html 